\title{
Luminescence from lanthanide(III) ions bound to the Glycocalyx of Chinese Hamster Ovary cells
}

Arppe-Tabbara, Riikka; Carro-Temboury, Miguel R; Hempel, Casper; Vosch, Tom; Sørensen, Thomas Just

Published in:

Chemistry - A European Journal

Link to article, DOI:

10.1002/chem.201802799

Publication date:

2018

Document Version

Peer reviewed version

Link back to DTU Orbit

Citation (APA):

Arppe-Tabbara, R., Carro-Temboury, M. R., Hempel, C., Vosch, T., \& Sørensen, T. J. (2018). Luminescence from lanthanide(III) ions bound to the Glycocalyx of Chinese Hamster Ovary cells. Chemistry - A European Journal, 24(46), 11885-11889. https://doi.org/10.1002/chem.201802799

\section{General rights}

Copyright and moral rights for the publications made accessible in the public portal are retained by the authors and/or other copyright owners and it is a condition of accessing publications that users recognise and abide by the legal requirements associated with these rights.

- Users may download and print one copy of any publication from the public portal for the purpose of private study or research.

- You may not further distribute the material or use it for any profit-making activity or commercial gain

- You may freely distribute the URL identifying the publication in the public portal 


\section{CHEMISTRY A European Journal}

\section{Accepted Article}

Title: Luminescence from lanthanide(III) ions bound to the Glycocalyx of Chinese Hamster Ovary cells

Authors: Riikka Arppe-Tabbara, Miguel R Carro-Temboury, Casper Hempel, Tom Vosch, and Thomas Just Sørensen

This manuscript has been accepted after peer review and appears as an Accepted Article online prior to editing, proofing, and formal publication of the final Version of Record (VoR). This work is currently citable by using the Digital Object Identifier (DOI) given below. The VoR will be published online in Early View as soon as possible and may be different to this Accepted Article as a result of editing. Readers should obtain the VoR from the journal website shown below when it is published to ensure accuracy of information. The authors are responsible for the content of this Accepted Article.

To be cited as: Chem. Eur. J. 10.1002/chem.201802799

Link to VoR: http://dx.doi.org/10.1002/chem.201802799 


\title{
Luminescence from lanthanide(III) ions bound to the Glycocalyx of Chinese Hamster Ovary cells
}

\author{
Riikka Arppe-Tabbara, ${ }^{[a]}$ Miguel R. Carro-Temboury, ${ }^{[a]}$ Casper Hempel, ${ }^{*[b]}$ Tom Vosch ${ }^{\star[a]}$ and Thomas \\ Just Sørensen*[a]
}

\begin{abstract}
Lanthanide(III) ions bind to the glycocalyx of Chinese Hamster Ovary $(\mathrm{CHO})$ cells and give rise to a unique luminescent fingerprint. Following direct excitation of terbium(III) or europium(III) ions in the visible part of the spectrum, we are able to collect emission spectra pixel-by-pixel in images of $\mathrm{CHO}$ cells. Following data analysis that removes the background signal, the fine structure of the europium(III) luminescence indicate that the lanthanide(III) ions are bound to a single structure of the $\mathrm{CHO}$ cell glycocalyx. This was deduced from the fact that the structure-sensitive emission spectrum of europium is unchanged throughout the investigated samples.
\end{abstract}

Lanthanide luminescence is unique as it manifests as distinct series of narrow emission lines with characteristic luminescence lifetimes. ${ }^{[1]}$ Either feature can be used to generate high contrast in fluorescence microscopy, ${ }^{[2]}$ which has been demonstrated for bioimaging using molecular probes based on green emitting terbium(III) or red emitting europium(III) ions. ${ }^{[3]}$ The probes are normally based on sensitized lanthanide luminescence, $\left.{ }^{[1,}, 3 f\right]$ where antenna chromophores are used to negate the low absorption coefficients of the lanthanide ions (Figure 1). While the design principle has been expanded to more lanthanide(III) ions ${ }^{[4]}$ it remains an unsolved challenge to create sensitizer chromophores that allow for excitation of lanthanide- based molecular probes using visible light. Here, we demonstrate that we can circumvent the sensitization step and use direct excitation of free europium(III) and terbium(III) ions and still record images and luminescence spectra from a biological sample by using a state-of-the-art fluorescence microscope. ${ }^{[2 a, 5]}$ Furthermore, we show that we can obtain background subtracted luminescence spectra from the individual pixels. Thereby, we are able to show that lanthanide(III) ions give rise to identical spectra in all pixels where they are present, suggesting that the ions exclusively bind to selected components of the glycocalyx of $\mathrm{CHO}$ cells.

Exploiting the unique luminescence features of lanthanides with narrow emission lines and long lifetimes was first demonstrated with labile complexes. ${ }^{[2 \mathrm{e}, 6]}$ Of note are the ultrasensitive DELFIA $®$ assay and time-gated high-throughput screening platforms based on the long luminescence lifetime of several lanthanide(III) ions. ${ }^{[6 e, 7]}$ Since the first demonstration, the focus has been on making molecular probes from kinetically inert complexes as they allow for clinical applications. ${ }^{[2 \mathrm{~d}, 3 \mathrm{a}, 3 \mathrm{e}, 8]}$

[a] Dr R. Arppe-Tabbara, Dr M.R. Carro-Temboury, Dr T. Vosch, Dr T. J. Sørensen

Nano-Science Center \& Department of Chemistry

University of Copenhagen

Universitetsparken 5, 2100 København Ø, Denmark

E-mail: tom@chem.ku.dk, tis@chem.ku.dk

[b] Dr C Hempel

Department of Micro- and Nanotechnology

Technical University of Denmark

Kgs Lyngby, Denmark

E-mail: casperhempel@gmail.com

One drawback of using sensitized lanthanide luminescence is that lanthanides are excited in the ultraviolet part of the spectrum. To enable excitation in the visible part of the spectrum direct excitation of forbidden $f-f$ transition can be used. Figure 1 shows the absorption spectra of a thioxanthone antenna chromophore and europium(III) and terbium(III) acetate. There is a 1000-fold difference in absorption efficiency which, however, is easily overcome by the efficiency of modern light sources and photodetectors-even spectrometers-with single molecule sensitivity. ${ }^{[2 a, 5]}$

Energy Transfer

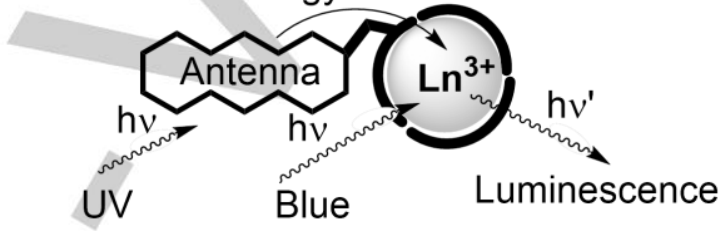

Excitation Excitation

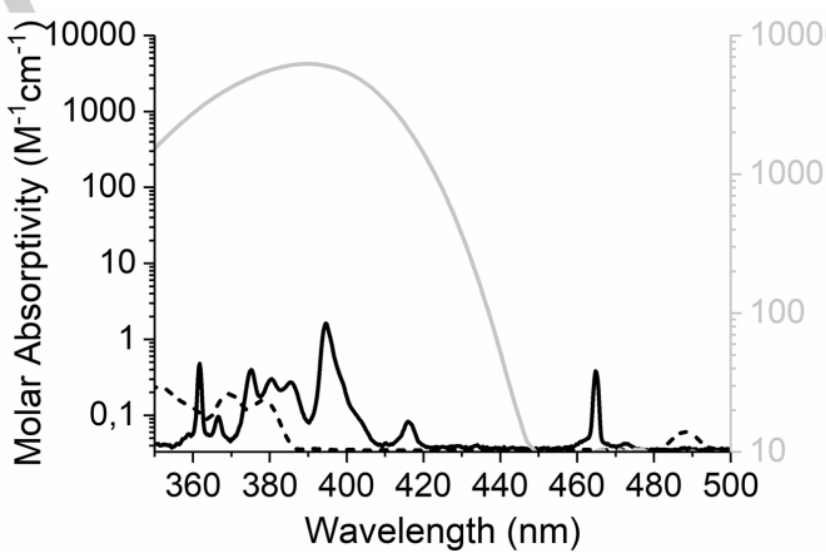

Figure 1. Top: Depiction of the antenna principle in lanthanide luminescence Bottom: Absorption spectra of europium(III) acetate (black, solid) and terbium(III) acetate (black, dash) in water, and an aza-thioxanthone dye (light grey, right $y$-axis) in methanol. ${ }^{[9]}$

While bioimaging following direct excitation of lanthanide ions has been demonstrated for kinetically inert lanthanide complexes, ${ }^{[10]}$ the use of free lanthanide(III) ion based probes for optical imaging has not been reported. This is not the case in bioimaging using transmission electron microscopy (TEM), ${ }^{[1]}$ where lanthanide(III) ions are well-known contrast agents. In TEM-based bioimaging, several protocols based on lanthanide(III) ions have been used for selective staining of glycocalyx. ${ }^{[11 a, 11 b, 12]}$ Optical bioimaging of glycocalyx primarily relies on specific antibodies or lectins that are detected with fluorochromes. ${ }^{[11 \mathrm{~b}, 13]}$ Here, we show that the lanthanide(III) ions that provide contrast in TEM, upon direct excitation can generate sufficient contrast in a confocal fluorescence microscope for luminescence spectra to be recorded in the individual 1 by 1 micrometer pixel. 
We chose to image formaldehyde fixed $\mathrm{CHO}$ cells following live cell staining (see Supporting Information for details). The cells were grown on cover slips and stained with a solution containing either $40 \mathrm{mM}$ lanthanide(III) acetate or a cocktail of lanthanide(III) acetate, F18, and Syto-9. The cells were then fixed with formaldehyde and then treated with SlowFade Gold antifade mounting medium, and mounted on a microscopy slide. Samples were prepared with terbium(III) acetate and europium(III) acetate. Spectrally resolved images were recorded of the four different samples types on a home-build confocal fluorescence microscope with single molecule detection capabilities. $^{[2 a, 5]}$

All samples were imaged in a 60-by-60-pixel array with a pixel size slightly exceeding 1 by 1 micrometer. To achieve sufficient signal a $1 \mathrm{~s}$ integration time per pixel was used, note that even in a state-of-the-art microscope acquiring these images takes up to 1 hour. The spectrally resolved images show that lanthanide(III) ions localize to the glycocalyx, as the spectra obtained from different regions of $\mathrm{CHO}$ cells only show the distinct fingerprint of lanthanide luminescence in the intercellular space. Figure 2 shows a representative image of a $\mathrm{CHO}$ cell stained with europium(III) acetate, F18 (a green membrane stain), and Syto-9 (a green nucleus stain) imaged following 465 $\mathrm{nm}$ excitation. F18 and Syto-9 allows the membrane and nucleus to be identified and spectra from individual pixels in selected regions of the cell are included in Figure 2. It is of note that no lanthanide(III) emission is seen inside the cells. The europium(III) emission spectra from the pixels containing europium(III) ions in Figure 2 are all similar. As the shape and relative peak ratios of the europium(III) spectrum depends on the symmetry and coordination chemistry, ${ }^{[1,14]}$ it will be different when in solution compared to when bound to the glycocalyx.
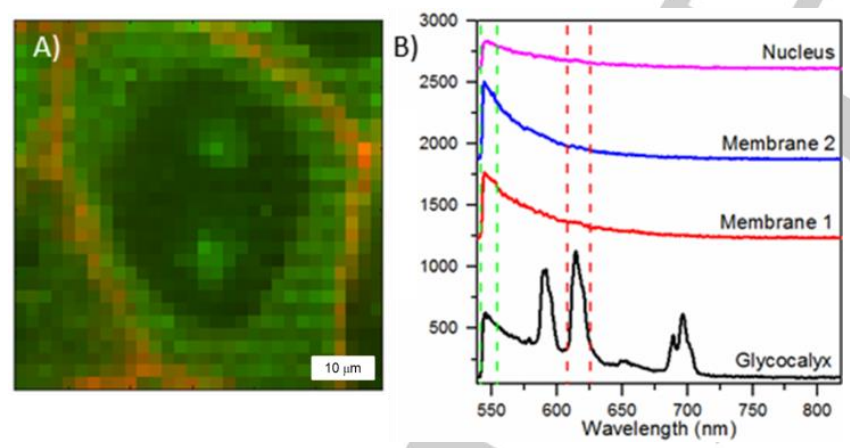

Figure 2. $\mathrm{CHO}$ cells stained with europium(III) acetate, F18 and Syto-9 imaged in a 60 by 60 micrometer area using a confocal scanning microscope equipped with a CCD spectrometer following $465 \mathrm{~nm}$ excitation. A, Pseudocolored image, $\mathrm{B}$, spectra from individual pixels from selected regions in the images corresponding to the cell nucleus, membrane and glycocalyx. The green and red dashed lines in the spectra illustrate the spectral regions used for pseudocoloring.

To investigate the sharp lines resulting from lanthanide luminescence in detail, we chose to resolve these using our previously introduced image analysis. ${ }^{[15]}$ This allows the fluorescent signal arising from lanthanide centered emission to be fully distinguished from auto-fluorescence and the signal arising from other fluorescent dyes. Figure 3 shows an image of $\mathrm{CHO}$ cells stained with europium(III), F18, and Syto-9. As expected, the two green-emitting dyes and the red emitting lanthanide(III) ion are excited at $465 \mathrm{~nm}$, and give rise to localized emission from the glycocalyx, membrane and nucleus, respectively. By plotting a pseudo-color image (Figure $3 \mathrm{~A}$ ) the individual cell components can be identified, even though probes cannot be differentiated. Figure $3 A$ shows that the membrane, the nuclei and the intercellular space are stained, and that the cytosol is dark. As we are able to uniquely identify the photons arising from europium(III) centered emission, ${ }^{[15]}$ we can conclude that the lanthanide luminescence gives rise to the orange-red areas in Figure $3 \mathrm{~A}$. The europium emission lines are clearly resolved in the total emission spectrum (from all pixels, Figure $3 \mathrm{~B})$. Image analysis allows us to resolve the counts originating from lanthanide centered emission, which gives rise to the background free image shown in Figure $3 \mathrm{C}$ that clearly shows that the europium(III) ions are localized in the extracellular space. This further allows the europium(III) emission spectrum to be studied in detail, as evident in the total emission spectrum shown in Figure 3D.

Figure 4 shows an image of $\mathrm{CHO}$ cells stained with terbium(III) acetate. The lanthanide(III) ions are excited directly at $488 \mathrm{~nm}$, and gives rise to localized emission from the glycocalyx. By plotting a pseudo-color image (Figure 4A) it is evident that contributions from auto-fluorescence originating from the cell plasma membrane give rise to contrast as well. In Figure 4B the narrow emission lines originating from terbium centered emission and a broad background signal from auto-fluorescence are shown. Using the data analysis algorithm pixel-by-pixel to uniquely identify the photons arising from terbium(III)-centered emission we obtain the image and spectrum shown in Figure 4C and $4 \mathrm{D} \cdot{ }^{[15]}$ Cursory inspection of the treated image shows that only the intercellular space contains terbium(III) ions. The emission spectra in Figure $4 \mathrm{~B}$ and 4D, from all pixels in the images, show that the lanthanide emission lines are resolved with a perfect contrast. ${ }^{[15]}$ Note that of the three peaks visible in the emission spectrum recorded over the entire spectrum (Figure 4B), only the most intense one is so intense that it is resolved by the algorithm that resolves the signal pixel-bypixel. $^{[15]}$ Thus, the images in Figure 2, 3 and 4 suggest that lanthanide(III) ions are found exclusively in the intercellular space and most likely in the glycocalyx of the $\mathrm{CHO}$ cells. Further examples can be found in the supporting information. 

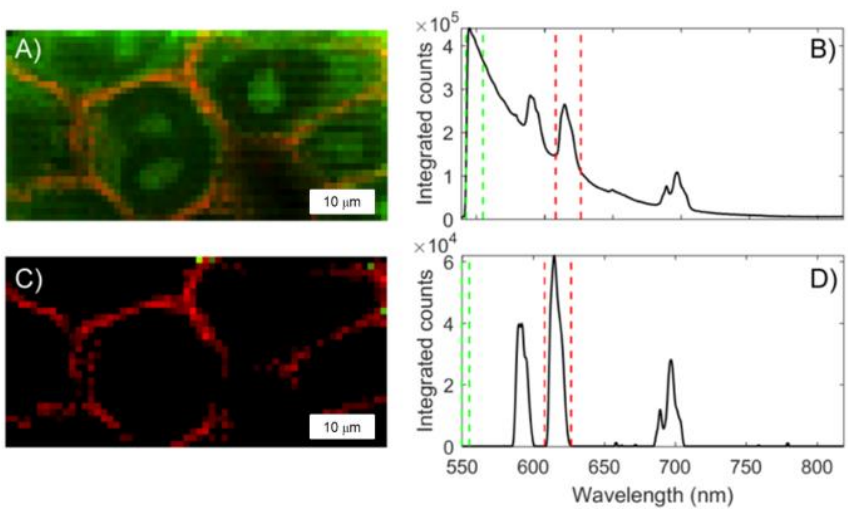

Figure 3.30 by 60 micrometer image of a single region of $\mathrm{CHO}$ cells stained with europium(III) acetate, F18 and Syto-9 imaged in a confocal scanning microscope equipped with a CCD spectrometer and following $465 \mathrm{~nm}$ excitation. A, pseudo-colored image. B, emission spectrum from all pixels in A. $C$, image of photons arising from lanthanide centered emission. $D$, emission spectrum from all pixels in $\mathrm{C}$. The green and red dashed lines in the spectra illustrate the spectral regions used for pseudocoloring.
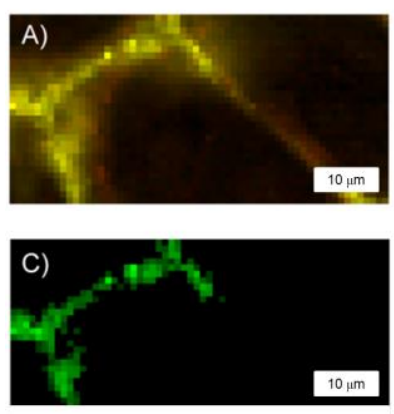
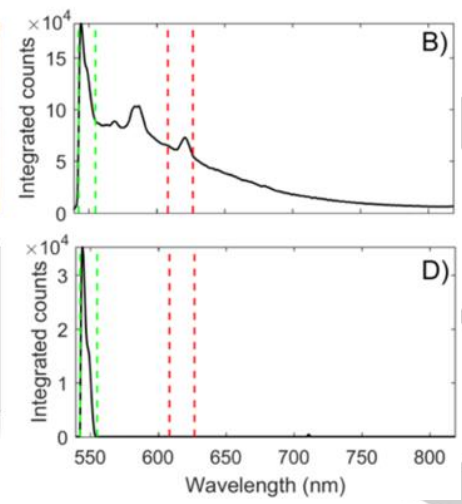

Figure 4.30 by 60 micrometer images of a single region of $\mathrm{CHO}$ cells stained with terbium(III) acetate imaged in a confocal scanning microscope equipped with a CCD spectrometer and following $488 \mathrm{~nm}$ excitation. A, pseudocolour image. $\mathrm{B}$, emission spectrum from all pixels in $\mathrm{A}$. $\mathrm{C}$, image of photons arising from lanthanide centered emission. D, emission spectrum from all pixels in $C$. The green and red dashed lines in the spectra illustrate the spectral regions used for pseudocolouring. For details on the image analysis, see SI.

We can now scrutinize the resolved lanthanide luminescence spectra from all pixels in all samples. As the spectra from all the europium(III) stained $\mathrm{CHO}$ cells are identical across all pixels and all images we can conclude that the geometry and donor atoms surrounding the europium ions are similar. ${ }^{[1]}$ The spectrum consists of narrow emission lines with a significant degree of fine structure. In the spectra obtained from the $\mathrm{CHO}$ cells, the ${ }^{5} \mathrm{D}_{0}$ to ${ }^{7} \mathrm{~F}_{4}$ band at $690 \mathrm{~nm}$ shows a particular degree of fine structure that allows us to conclude that all the emitting europium(III) species are similar if not identical. This leads to a hypothesis where all the lanthanide ions predominantly bind to a single, specific structure in the $\mathrm{CHO}$ cell glycocalyx. Based on the studies performed using TEM and the fact that lanthanide ions prefer hard anionic ligands, ${ }^{[11 a]}$ we propose that these structures are anionic motifs in the glycocalyx. The binding pockets exhibited by the different anionic sugars are significantly different, indicating that the lanthanide ions must be bound either to hyaluronic acid, heparan sulfate, chondroitin sulfate or sialic acid residues. At this point we cannot differentiate which, but based on the relatively weak symmetry-dependent $580 \mathrm{~nm}$ band (the untreated spectra are available as supporting information) in the spectrum we can conclude that the europium(III) ions are located in identical, symmetric binding pockets. $^{[1]}$

To substantiate the claim that the lanthanide(III) ions are bound to a single structural motif of the glycocalyx, we produced control samples of europium(III) acetate with and in the absence of $\mathrm{CHO}$ cells. If either control gave rise to similar emission spectra, the claim could be disproved. Figure 5 shows the resolved emission spectra normalized to the $590 \mathrm{~nm}$ emission peak from samples with $\mathrm{CHO}$ cells stained with europium(III) along with spectra from control samples with europium(III) acetate dried in the formaldehyde with and without the SlowFade Gold mounting medium. Cursory inspection of Figure 5 shows that the shapes and peak ratios of the resolved spectra from the control samples are different from each other and from the spectrum from $\mathrm{CHO}$ cells. This confirms that the lanthanide ions are indeed bound to the $\mathrm{CHO}$ cell glycocalyx, not by the fixing or mounting agents.

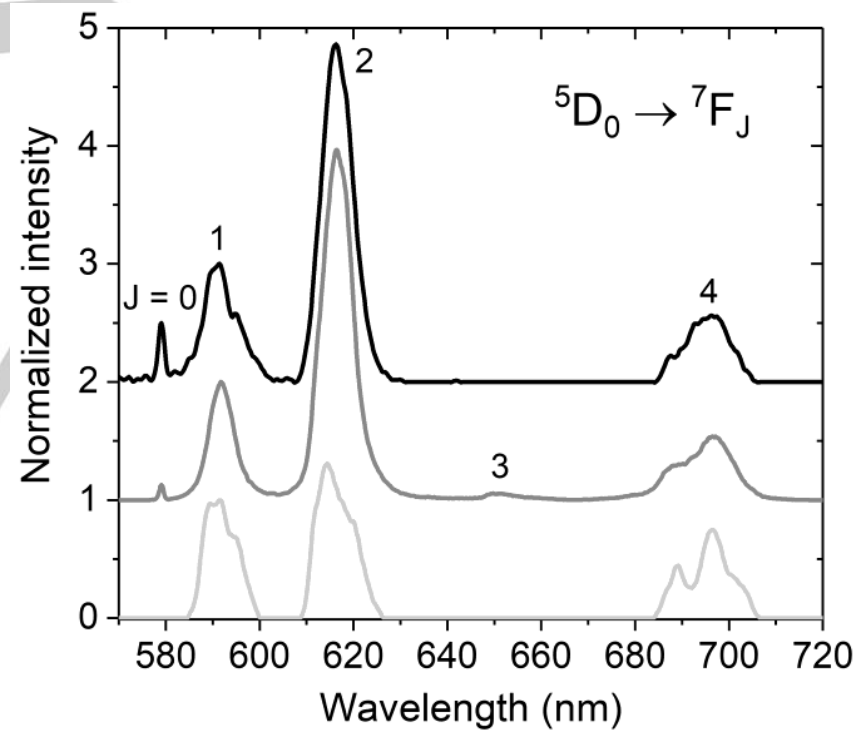

Figure 5 Europium(III) emission spectra from images recorded samples with europium(III) acetate in formaldehyde with (black) and without (grey) slow fade gold, and bound to the glycocalyx of $\mathrm{CHO}$ cells (light grey) and analyzed using the signal resolving algorithm. The spectra have been normalized to the 590 nm peak.

In summary, we have shown that it is possible to use direct excitation of terbium(III)- and europium(III) ion to obtain high contrast images of $\mathrm{CHO}$ cells. We used a home-built fluorescence microscope that allowed us to exploit the unique features of europium(III) luminescence to show that lanthanide(III) ions were present exclusively in the glycocalyx of 
the $\mathrm{CHO}$ cells. The distinct europium(III) spectrum was used further, to show that the lanthanide ions all bind to similar ligands, which we suggest is one specific component of the glycocalyx. As we show that the highly inefficient direct excitation of low absorbance $f-f$ transitions can be used for spectrally resolved fluorescence microscopy, we conclude that lanthanide luminescence following direct excitation should be accessible using conventional laser lines in standard fluorescent microscopes.

\section{Acknowledgements}

We thank bio-SYNergy University of Copenhagen's Excellence Programme for Interdisciplinary Research, Villum Fonden, the "Center for Synthetic Biology" at Copenhagen University funded by the UNIK research initiative of the Danish Ministry of Higher Education \& Science (09-065274), and the Danish Council of Independent Research (DFF-1323-00352 and DFF-6110-00554) for support.

Keywords: Lanthanide Luminescence • Spectroscopy • Coordination Chemistry $\bullet$ Microscopy $\bullet$ Glycocalyx

[1] P. Hänninen, H. Härmä, Lanthanide Luminescence, Vol. 7, Springer, Heidelberg, 2011.

[2] a) Z. Liao, M. Tropiano, K. Mantulnikovs, S. Faulkner, T. Vosch, T. Just Sørensen, Chem. Comm. 2015, 51, 2372-2375; b) Z. Liao, M. Tropiano, S. Faulkner, T. Vosch, T. J. Sørensen, RSC Adv. 2015, 70282-70286; c) A. Grichine, A. Haefele, S. Pascal, A. Duperray, R. Michel, C. Andraudd, O. Maury, Chem. Sci. 2014, 5, 3475; d) A. Beeby, S. W. Botchway, I. M. Clarkson, S. Faulkner, A. W. Parker, D. Parker, J. A. G. Williams, J. Photochem. Photobi. B-Bio. 2000, 57, 83-89; e) G. Vereb, E. Jares-Erijman, P. R. Selvin, T. M. Jovin, Biophys J 1998, 74, 22102222.

[3] a) C. P. Montgomery, B. S. Murray, E. J. New, R. Pal, D. Parker, Acc Chem Res 2009, 42, 925-937; b) Y. Bretonniere, M. J. Cann, D. Parker, R. Slater, Chem. Comm. 2002, 1930-1931; c) A. T. Bui, A. Grichine, A. Duperray, P. Lidon, F. Riobe, C. Andraud, O. Maury, J. Am. Chem. Soc. 2017, 139, 7693-7696; d) A. Picot, A. D'Aléo, P. L. Baldeck, A. Grichine, A. Duperray, C. Andraud, O. Maury, J. Am. Chem. Soc. 2008, 130, 1532-1533; e) E. G. Moore, A. P. Samuel, K. N. Raymond, Acc Chem Res 2009, 42, 542-552; f) J. C. Bunzli, Chem Rev 2010, 110, 27292755.

[4] a) A. T. Bui, M. Beyler, A. Grichine, A. Duperray, J. C. Mulatier, Y. Guyot, C. Andraud, R. Tripier, S. Brasselet, O. Maury, Chem. Comm. 2017, 53, 6005-6008; b) A. T. Bui, A. Grichine, S. Brasselet, A. Duperray, C. Andraud, O. Maury, Chem. Eur. J. 2015, 21, 1775717761.

[5] Z. Liao, E. N. Hooley, L. Chen, S. Stappert, K. Müllen, T. Vosch, J. Am. Chem. Soc. 2013, 135, 19180-19185.

[6] a) P. R. Selvin, J. E. Hearst, Proc. Natl Ac. Sci. USA 1994, 91, 1002410028; b) I. Hemmilá, V.-M. Mukkala, Critical Rev. Clinical Lab. Sci. 2008, 38, 441-519; c) I. Hemmilä, S. Dakubu, V.-M. Mukkala, H. Siitari, T. Lövgren, Anal Biochem. 1984, 137, 335-343; dE. Soini, I. Hemmila Clin Chem 1979, 25, 353-361; e) J. M. Zwier, N. Hildebrandt, in Reviews in Fluorescence 2016 (Ed.: C. D. Geddes), Springer, 2017, pp 17-43; f) M. Latva, H. Takalo, V.-M. Mukkala, C. Matachescu, J. C Rodriguez-Ubis, J. Kankare, J. Luminesc. 1997, 75, 149-169; g) P. R. Selvin, Annu Rev Biophys Biomol Struct 2002, 31, 275-302.
[7] a) N. Hildebrandt, K. D. Wegner, W. R. Algar, Coord Chem Rev 2014 273-274, 125-138; b) H. S. Afsari, M. Cardoso Dos Santos, S. Lindén, T. Chen, X. Qiu, P. M. P. van Bergen en Henegouwen, T. L. Jennings, K. Susumu, I. L. Medintz, N. Hildebrandt, L. W. Miller, Science Advances 2016, 2; c) M. Cardoso Dos Santos, J. Goetz, H. Bartenlian, K. L. Wong, L. J. Charbonnière, N. Hildebrandt, Bioconjugate chemistry 2018, 29, 1327-1334; d) J. M. Zwier, H. Bazin, L. Lamarque, G. Mathis Inorg. Chem. 2014, 53, 1854-1866.

[8] a) A. D. Sherry, P. Caravan, R. E. Lenkinski, Journal of Magnetic Resonance Imaging 2009, 30, 1240-1248; b) D. Kovacs, X. Lu, L. S Meszaros, M. Ott, J. Andres, K. E. Borbas, J. Am. Chem. Soc. 2017, 139, 5756-5767; c) T. J. Sørensen, A. M. Kenwright, S. Faulkner, Chem. Sci. 2015, 6, 2054-2059; d) U. Cho, D. P. Riordan, P. Ciepla, K. S. Kocherlakota, J. K. Chen, P. B. Harbury, Nat Chem Bio 2017, 14, 15-21

[9] a) A. K. R. Junker, T. J. Sørensen, Meth. Appl. Fluoresc. 2018, 6, 014002; b) R. Arppe, N. Kofod, A. K. R. Junker, L. G. Nielsen, E. Dallerba, T. Just Sørensen, Eur J Inorg Chem 2017, 2017, 5246-5253.

[10] B. S. Murray, E. J. New, R. Pal, D. Parker, Org Biomol Chem 2008, 6, 2085-2094.

[11] a) K. P. Arkill, C. R. Neal, J. M. Mantell, C. C. Michel, K. Qvortrup, J. Rostgaard, D. O. Bates, C. Knupp, J. M. Squire, Microcirculation 2012 19, 343-351; b) S. Reitsma, D. W. Slaaf, H. Vink, M. A. van Zandvoort M. G. oude Egbrink, Pflugers Arch 2007, 454, 345-359; c) T. S. LEESON, G. W. HIGGS, Histochemical Journal 1982, 14, 553-560.

[12] a) R. C. Wagner, S. C. Chen, Journal of Histochemistry \& Cytochemistry 1990, 38, 275-282; b) H. Fehrenbach, A. Schmiedl, F. Brasch, J. Richter, Journal of Microscopy 1994, 174, 207-223; c) A. Schmiedl, F. Bach, H. Fehrenbach, P. A. Schnabel, J. Richter, The Anatomical Record 1995, 243, 496-508.

[13] S. M. Haslam, S. Julien, J. M. Burchell, C. R. Monk, A. Ceroni, O. A Garden, A. Dell, Immunology And Cell Biology 2008, 86, 564.

[14] L. G. Nielsen, A. K. R. Junker, T. J. Sørensen, Dalton transactions 2018.

[15] M. R. Carro-Temboury, R. Arppe, C. Hempel, T. Vosch, T. Just Sørensen, Plos One 2017, 12, e0189529. 
Entry for the Table of Contents (Please choose one layout)

Layout 1:

\section{COMMUNICATION}

State-of-the-art microscopy allows us to record lanthanide luminescence spectra in every pixel when imaging cells. Here, we use this information to show that lanthanide(III) ions bind to a specific part of the glycocalyx of Chinese hamster Ovary or $\mathrm{CHO}$ cells.

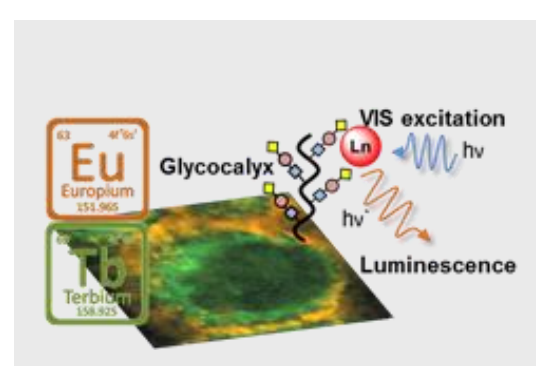

Riikka Arppe-Tabbara, Miguel R. CarroTemboury, Casper Hempel, * Tom Vosch $^{\star}$ and Thomas Just Sørensen* Page No. - Page No.

Luminescence from lanthanide(III) ions bound to the Glycocalyx of Chinese Hamster Ovary cells 